\title{
Clinical features and risk factors of neurological involvement in Sjögren's syndrome
}

\author{
Wenjing Ye ${ }^{1,2}$, Siyan Chen ${ }^{3}$, Xinshi Huang ${ }^{1}$, Wei Qin ${ }^{1}$, Ting Zhang ${ }^{1}$, Xiaofang Zhu' ${ }^{1}$ Xiaochun Zhu' , \\ Chongxiang Lin ${ }^{4^{*}}$ and Xiaobing Wang ${ }^{1 *}$
}

\begin{abstract}
Background: To investigated distinct manifestations of Sjögren's syndrome (SS) patients with neurological complications and the potential risk factors associated with neurological complications in SS, and to produce a disease evaluation and neurological involvement prediction for SS.

Methods: 566 patients who fulfilled the 2002 classification criteria for SS from the Rheumatology Department of the First Affiliated Hospital of Wenzhou Medical University were included in the cross-sectional study. Clinical, immunological and histological characteristics were surveyed, and potential risk factors for neurological complications were examined by multivariate analysis.

Results: Among 566 SS patients, 184 (32.5\%) patients had neurological involvement, with more than 10\% got limbs pain, limbs numbness and cerebral infarction, respectively. Of these 184 SS patients with neurological complications, secondary SS (SSS) patients had a higher prevalence of peripheral nervous system (PNS) involvement than primary SS (pSS) patients (31.1 vs. 19\%). And sSS patients showed higher total ESSPRI score and higher prevalence of xerostomia and low C3, C4 levels with more liver, articular involvement and saliva gland atrophy, and more severe lymphocyte infiltration in salivary glands than pSS patients. As for the specific factors associated with neurological involvement, low C3 level were found to be significant in pSS or sSS patients who were younger 50 year old, and ANA positivity, cardiac involvement, saliva gland atrophy were demonstrated to be associated in elder pSS patients. And xerophthalmia was found to be associated in sSS patients.
\end{abstract}

Conclusion: Low complement (C3) levels, xerophthalmia, ANA positive, cardiac involvement and labial salivary gland histological result were good ways to predict neurological complications in different subgroups of SS, which might provide insight into better clinical decision-making, especially at early stages of the disease.

Keywords: Sjögren's syndrome, Cross-sectional study, Neurological complication, Risk factor, Logistic model

\section{Background}

Sjögren's syndrome (SS) is a chronic autoimmune disease characterized by autoantibody secretion and lymphocytic infiltrates of exocrine glands leading to xerophthalmia and xerostomia [1]. Sjögren's syndrome is considered

\footnotetext{
*Correspondence: 6785069@qq.com; gale820907@163.com

${ }^{1}$ Rheumatology Department, The First Affiliated Hospital of Wenzhou

Medical University, Wenzhou, China

${ }^{4}$ Stomatological Department, The First Affiliated Hospital of Wenzhou

Medical University, Wenzhou, China

Full list of author information is available at the end of the article
}

secondary (sSS) when it presents with associated autoimmune disorders, otherwise it's considered primary Sjögren's syndrome (pSS) [2]. It impacts $0.1-0.6 \%$ of the general adult population with a female to male ratio of 9:1 [3]. Besides dry eyes and dry mouth, pSS covers a broad spectrum of extraglandular clinical presentations, including vasculitis, arthralgia, renal tubular acidosis, pulmonary involvement, immunological abnormalities and neuropathy $[4,5]$.

Neurological disorders of SS including peripheral neuropathy and central neuropathy were investigated since 
the 1980s [6], however, their prevalence are inconstant in different studies. It's documented that $10-20 \%$ of SS patients had peripheral nervous system (PNS) involvement [7]. However, the prevalence of central nervous system (CNS) manifestations of SS ranged from 2.5 to $60 \%$ [8], which may due to the lack of unified definition of CNS involvement in SS $[9,10]$. Therefore, CNS and PNS complications are common but varied in SS. Furthermore, the association between clinical/serological manifestations and nervous system involvement remains unclear. To date no seroimmunological profiles had been shown as pathognomonic neither for neurological involvement in SS. The aim of this study was to evaluate the prevalence and symptoms of neurological complications in a population of patients with SS and to investigate the potential risk factors for neurological involvement to facilitate a comprehensive disease evaluation and prognosis prediction for Chinese SS patients.

\section{Methods}

\section{Study population and clinical data}

A total of 566 patients who fulfilled the 2002 classification criteria [8] for SS from the Rheumatology department of the First Affiliated Hospital of Wenzhou Medical University between January 1, 2013 and February 28, 2017 were enrolled in this study. The study was approved by the Ethical Committee of the First Affiliated Hospital of Wenzhou Medical University (approval \# 16024). The study design conformed to current National Health and Family Planning Commission of China ethical standards, with written informed consent provided by all patients. sSS patients were diagnosed with systemic lupus erythematosus [11], systemic sclerosis [12], rheumatoid arthritis [13], inflammatory myopathies [14], mixed connective tissue disease [15], or primary biliary cirrhosis [16] according to existing classification criteria used in clinical practice. Clinical data as age of disease onset, age at diagnosis, the duration of the disease, oral and ocular dryness, constitutional symptoms, joints, skin, pulmonary, kidney, vasculitis, gastrointestinal tracts and endocrine involvement were collected.

\section{Assessment of nervous system involvement}

Peripheral neuropathy includes painful sensory neuropathy, sensory ataxic neuropathy, pure sensory trigeminal neuropathy, axonal sensorimotor polyneuropathy, mononeuritis multiplex, multiple cranial neuropathies, radiculoneuropathy and autonomic neuropathy. Involvement of peripheral nervous system was diagnosed by medical history, physical examination and electromyography. Involvement of central nervous system was confirmed by case history brain computed tomography, cranial MRI, electromyography and psychiatric records. And the presentations we collect including headache, dizziness, physical pain, epilepsy, cognitive disorder, disturbance of consciousness, emotional problem, cerebral hemorrhage, cerebral infarction, demyelination, vascular stenosis and occlusion, aging brain, cerebral tumor, leukoaraiosis and nerve injury. Depression was diagnosed by psychiatrists.

\section{Assessment of exocrine gland involvement and disease activity}

All patients underwent a thorough review of medical history, physical examination and a series of assessments to evaluate the systemic condition. Xerostomia was diagnosed by testing timed whole unstimulated salivary flow and the histopathological result of labial salivary gland biopsy (LSGB). A lymphocytic focus score of $\geq 1$ as a positive biopsy with more than 50 lymphocytes per $4 \mathrm{~mm}^{2}$, based on the classification described previously [17]. Xerophthalmia was confirmed by an ophthalmologist by examining Schirmer's test [16], breakup time of tear film [18] and cornea fluorescent pigmentation [19].

Besides routine blood test, immunological tests including ANA, anti-Ro antibodies, anti-SSA antibodies, antiSSB antibodies, rheumatoid factor, C3, C4, IgG, IgA, and IgM levels were performed using commercial techniques. Additionally, all patients were tested for acute inflammatory factors comprising erythrocyte sedimentation rate (ESR), and C-reactive protein (CRP) levels.

Disease manifestations were scored with the ESSDAI summating the scores achieved per organ domain. The ESSDAI scores 12 organ domains on the severity of involvement, ranging from 0 to 3 points. And each patient was asked to assess the severity of dryness, fatigue, and pain over the preceding 2 weeks, each on a 10-point Likert scale. The average of the three items was regarded as ESSPRI value [20].

\section{Statistical analysis}

Continuous data were expressed as mean \pm standard deviation, and counts and percentages were indicated for the categorical variables. Nonparametric Wilcoxon signed-rank test was used for paired comparison and Fisher's exact tests used for categorical data as appropriate. To examine correlations between risk factors and neuropathy, univariate analyses were used, firstly based on biological plausibility and literature review. Variables with a $P<0.05$ in univariate analysis were then included in a multivariate analysis using logistic regression. Statistical significance was set at $P<0.05$. All analyses were conducted using R v3.3.2 statistical software packages. 


\section{Results}

The characteristics of Sjögren's syndrome patients with or without neurological involvement

The patient cohort comprised 566 individuals, with 415 pSS and 151 sSS patients. Demographic, clinical, histological, immunological, inflammatory features and outcome measures data collected from 415 pSS were presented in Table 1. The female to male ratio is 10:1. 290 (69.88\%) pSS patients had no neurological involvements and 125 (30.12\%) with neurological involvement. Most patients presented to the hospital in their 50s for the first interview, with an average disease course of approximate 4 years. Compared with pSS patients without neurological involvement, those with neurological involvement showed much higher total ESSDAI score with more cardiac and constitutional domain involvements, but less glandular domain involvement. It is consistent with a lower prevalence of xerostomia in this subgroup. However, no significant difference in total ESSPRI scores was found between the two groups. pSS patients with neurological involvement showed a higher prevalence of ANA antibody and hypergammaglobulinemia, but reduced level of $\mathrm{C} 3(P<0.05)$. No significant differences in Anti-SSA/SSB positive was found between the two subgroups $(P>0.05)$. Although pSS patients with neurological involvement seemed to use glucocorticoid more often with higher dose and longer duration, the differences didn't reach to the statistical significance $(P<0.05$, Table 1$)$. There are $92(60.93 \%)$ sSS patients had no neurological involvements and $59(39.07 \%)$ with neurological

Table 1 Demographic, clinical, histological, immunological, inflammatory features and outcome measures of Chinese primary Sjögren's syndrome patients with or without neurological involvement

\begin{tabular}{|c|c|c|c|}
\hline & $\begin{array}{l}\text { Without NP involvement } \\
290\end{array}$ & $\begin{array}{l}\text { With NP involvement } \\
125\end{array}$ & $P$ value \\
\hline Age (years) & $51.50 \pm 13.73$ & $52.81 \pm 14.95$ & 0.387 \\
\hline Sex, female, n (\%) & $28(9.66 \%)$ & $16(12.80 \%)$ & 0.34 \\
\hline Disease duration (months) & $3.99 \pm 6.01$ & $4.30 \pm 5.57$ & 0.642 \\
\hline Hypertension, n (\%) & $57(19.66 \%)$ & $29(23.20 \%)$ & 0.414 \\
\hline Diabetes mellitus, n (\%) & $22(7.59 \%)$ & $12(9.60 \%)$ & 0.493 \\
\hline Hyperlipemia, n (\%) & $114(39.31 \%)$ & $37(29.60 \%)$ & 0.059 \\
\hline Xerostomia, n (\%) & $237(81.72 \%)$ & $88(70.40 \%)$ & 0.010 \\
\hline Xerophthalmia, n (\%) & $80(27.59 \%)$ & $44(35.20 \%)$ & 0.120 \\
\hline ESSDAI & $6.60 \pm 2.22$ & $8.81 \pm 3.45$ & $<0.001$ \\
\hline ESSPRI & $2.87 \pm 1.51$ & $2.97 \pm 1.46$ & 0.459 \\
\hline Constitutional domain involvement & $65(22.41 \%)$ & $42(33.60 \%)$ & 0.017 \\
\hline Glandular domain involvement & $189(65.17 \%)$ & $47(37.60 \%)$ & $<0.001$ \\
\hline Cardiac involvement, n (\%) & $133(45.86 \%)$ & $77(61.60 \%)$ & 0.003 \\
\hline LSGB, Lymphocytic focus $\geq 1$ & $192(66.21 \%)$ & $76(60.80 \%)$ & 0.291 \\
\hline ANA positivity, $\mathrm{n}(\%)$ & $254(87.59 \%)$ & $120(96.00 \%)$ & 0.008 \\
\hline Anti-SSA/Ro60 positive, n (\%) & $214(75.09 \%)$ & $97(78.23 \%)$ & 0.494 \\
\hline Anti-Ro52 positive, n (\%) & $177(62.11 \%)$ & $79(63.20 \%)$ & 0.833 \\
\hline Anti-SSB positive, $\mathrm{n}(\%)$ & $134(47.02 \%)$ & $50(40.00 \%)$ & 0.188 \\
\hline Hypergammaglobulinemia (> $16 \mathrm{~g} / \mathrm{L}$ ), n & $186(64.14 \%)$ & $120(100.00 \%)$ & $<0.001$ \\
\hline Low C3 level $(<0.9 \mathrm{~g} / \mathrm{L})$ & $90(31.03 \%)$ & $54(46.15 \%)$ & 0.004 \\
\hline Low C4 level $(<0.1 \mathrm{~g} / \mathrm{L})$ & $11(4.17 \%)$ & $6(5.31 \%)$ & 0.624 \\
\hline RF positive, n (\%) & $178(61.38 \%)$ & $59(52.21 \%)$ & 0.093 \\
\hline $\mathrm{ESR}(\mathrm{mm} / \mathrm{h})$ & $35.71 \pm 23.84$ & $32.43 \pm 25.31$ & 0.232 \\
\hline $\mathrm{CRP}(\mathrm{mg} / \mathrm{L})$ & $10.23 \pm 21.68$ & $12.76 \pm 24.51$ & 0.325 \\
\hline Glucocorticoid use & $171(58.97 \%)$ & $81(66.39 \%)$ & 0.158 \\
\hline Moderate to high dose & $46(15.86 \%)$ & $24(19.20 \%)$ & 0.405 \\
\hline Continuous use for $>1$ year & $81(27.93 \%)$ & $38(30.40 \%)$ & 0.610 \\
\hline
\end{tabular}

Disease duration measured from the day diagnosed; moderate- to high-dose glucocorticoid: > $1 \mathrm{mg} / \mathrm{kg} / \mathrm{d}$ prednisone; glucocorticoid use at any time for $>2 \mathrm{week}$ NP neurological, ESSDAI European League Against Rheumatism Sjögren's syndrome disease activity index, ESSPRI European League Against Rheumatism Sjögren's syndrome patient reported index, ANA antinuclear antibodies, C3 complement component 3, C4 complement component 4, CRP C-reactive protein, ESR erythrocyte sedimentation rate, $L S G B$ labial salivary gland biopsy, $R F$ rheumatoid factor 
involvement. The comparison between with and without neurological involvement were not analyzed as sSS cases had different primary diseases with distinctive etiology and mechanism.

\section{The characteristics of neurological involvement in Sjögren's syndrome patients}

As shown in Table 2, the various manifestations of neurological involvements were categorized into five subgroups: PNS presentations, neuropsychiatric symptoms, unspecific neurological complaints, paroxysmal disease, and CNS radiological results. The prevalence of limbs pain, limbs numbness and cerebral infarction were all higher than $10 \%$. Meanwhile, the occurrences of trigeminal neuralgia, epilepsy, cerebral hemorrhage, angiostegnosis, cerebral tumor and leukoaraiosis were comparatively low. Of note, there were 39 patients with neurological involvement had sleep problem as well.

\section{Subtypes of neurological involvement in Sjögren's syndrome patients}

Among 415 pSS patients, there were 125 of them had neurological involvement with 79 PNS involvement and

Table 2 Features of neurological involvement in Sjögren's syndrome patients

\begin{tabular}{lcl}
\hline Neurological involvement & Numbers & Percentage (\%) \\
\hline PNS presentations & 35 & 0.190 \\
Limbs pain & 25 & 0.136 \\
Limbs numbness & 8 & 0.043 \\
Anaesthesia & 1 & 0.005 \\
Trigeminal neuralgia & & \\
Neuropsychiatric symptoms & 10 & 0.054 \\
Depression & 4 & 0.022 \\
Cognitive dysfunction & & \\
Unspecific neurological complaints & 15 & 0.082 \\
Headache & 16 & 0.087 \\
Dizziness & 11 & 0.060 \\
Muscular weakness & & \\
Paroxysmal disease & 3 & 0.016 \\
Epilepsy & & \\
CNS radiological results & 2 & 0.011 \\
Cerebral hemorrhage & 35 & 0.190 \\
Cerebral infarction & 5 & 0.027 \\
Demyelination & 3 & 0.016 \\
Angiostegnosis & 11 & 0.060 \\
Aging brain & 3 & 0.016 \\
Cerebral tumor & 35016 \\
Leukoaraiosis & & \\
\hline PNS peripheranervous system, & & \\
\hline
\end{tabular}

PNS peripheral nervous system, CNS central nervous system
63 CNS involvement. 59 out of 151 sSS patients got neurological involvement. And sSS had a higher prevalence of PNS involvement than pSS (31.13 vs. $19.04 \%, P<0.05)$, although no significant difference in CNS involvement (13.91 vs. $15.18 \%)$. Most of the sSS cases with neurological involvement were secondary to systemic lupus erythematosus (SLE) or rheumatoid arthritis (RA) (Table 3).

Table 3 Subtypes of neurological involvement in Sjögren's syndrome patients

\begin{tabular}{llc}
\hline & PNS involvement & CNS involvement \\
\hline pSS $(n=125)$ & 79 & 63 \\
sSS $(n=59)$ & 47 & 21 \\
Rheumatoid arthritis & 13 & 5 \\
Systemic lupus erythematosus & 28 & 13 \\
Systemic sclerosis & 1 & 0 \\
Dermatomyositis/myositis & 3 & 0 \\
Primary biliary cirrhosis & 0 & 3 \\
Mixed connective tissue & 1 & 1 \\
$\quad$ disease & &
\end{tabular}

Table 4 Analysis of multiple features of 184 Chinese Sjögren's syndrome patients with neurological involvement

\begin{tabular}{lllr}
\hline & pSS & sSS & P value \\
& $\mathbf{1 2 5}$ & $\mathbf{5 9}$ & \\
\hline Age (years) & $52.81 \pm 14.95$ & $47.93 \pm 16.22$ & 0.084 \\
Sex, female, n (\%) & $109(87.20 \%)$ & $56(94.90 \%)$ & 0.126 \\
Disease duration (months) & $51.57 \pm 66.84$ & $46.07 \pm 68.73$ & 0.955 \\
Xerostomia, n (\%) & $88(70.40 \%)$ & $50(84.70 \%)$ & 0.036 \\
Xerophthalmia, $\mathrm{n}(\%)$ & $44(35.20 \%)$ & $22(37.30 \%)$ & 0.783 \\
ESSDAl & $7.84 \pm 2.83$ & $8.81 \pm 3.45$ & 0.129 \\
ESSPRI & $2.77 \pm 1.34$ & $3.38 \pm 1.63$ & 0.038 \\
Liver dysfunction, $\mathrm{n}(\%)$ & $41(33.60 \%)$ & $28(49.10 \%)$ & 0.047 \\
Articular involvement, $\mathrm{n}(\%)$ & $46(36.80 \%)$ & $40(67.80 \%)$ & $<0.001$ \\
LSGB, lymphocytic focus $\geq 1$ & $76(60.80 \%)$ & $50(84.70 \%)$ & 0.001 \\
Saliva gland atrophy & $78(70.30 \%)$ & $46(86.80 \%)$ & 0.021 \\
Albumin levels (g/L) & $37.35 \pm 5.73$ & $34.12 \pm 5.94$ & $<0.001$ \\
Low C3 level (<0.9 g/L) & $54(46.20 \%)$ & $36(65.50 \%)$ & 0.018 \\
Low C4 level (<0.1 g/L) & $6(5.30 \%)$ & $11(20.80 \%)$ & 0.005 \\
Antiphospholipid syndrome & 0 & $6(10.17 \%)$ & $<0.001$ \\
\hline
\end{tabular}

$N P$ neurological, ESSDAl European League Against Rheumatism Sjögren's syndrome disease activity index, ESSPRI European League Against Rheumatism Sjögren's syndrome patient reported index, ANA antinuclear antibodies, C3 complement component 3, C4 complement component 4, CRP C-reactive protein, ESR erythrocyte sedimentation rate, LSGB labial salivary gland biopsy, RF rheumatoid factor 
Features of Sjögren's syndrome patients with neurological involvement

As shown in Table 4, sSS showed higher total ESSPRI score and higher prevalence of xerostomia and low C3, C4 levels with more liver, articular involvement and saliva gland atrophy, while pSS had a higher level of albumin. And sSS showed more severe lymphocyte infiltration in salivary glands than pSS. There were 6 antiphospholipid syndrome cases were found in sSS but none in pSS.

\section{Specific factors associated with neurological involvement in Sjögren's syndrome}

A series of features commonly used in clinical practice were selected first by univariate analysis and then logistic regression analysis as potential risk factors for neurological involvement in SS. By univariate analysis a series of variables were found to be associated with neurological involvement, as shown in Table 5. And their independent risks for neurological involvement were further tested by multivariate analysis. As age is a key factor on neurological involvement, we did a stratification analysis

Table 5 Univariate and multivariate analysis of factors associated with neurological involvement in primary and secondary Sjögren's syndrome

\begin{tabular}{lll}
\hline $\begin{array}{l}\text { Independent } \\
\text { variables }\end{array}$ & \multicolumn{2}{l}{$\begin{array}{l}\text { Univariate analysis } \\
\text { OR }(95 \% \mathrm{Cl}) \boldsymbol{P} \text { value }\end{array}$} \\
\cline { 2 - 3 } & pSS & sSS \\
\hline Age $<50$ & & \\
Sex (male) & $4.55(1.27,16.29) 0.020$ & - \\
ESSDAl & $1.47(1.26,1.72)<0.001$ & $1.27(1.05,1.53) 0.0136$ \\
Xerophthalmia, $\mathrm{n}(\%)$ & & $3.25(1.03,10.23) 0.0440$ \\
Low C3 level $(<0.9 \mathrm{~g} / \mathrm{L})$ & $2.10(1.06,4.13) 0.032$ & $3.83(1.31,11.24) 0.0143$ \\
Low C4 level $(<0.1 \mathrm{~g} / \mathrm{L})$ & - & $3.50(1.02,12.00) 0.0463$ \\
RF positive, $\mathrm{n}(\%)$ & - & $0.32(0.12,0.87) 0.0251$ \\
ESR (mm/h) & $0.98(0.96,1.00) 0.038$ & - \\
Age $\geq 50$ & & \\
Constitutional domain & $1.96(1.08,3.55) 0.027$ & - \\
involvement & & \\
ESSDAl & $1.52(1.34,1.72)<0.001$ & $1.44(1.16,1.80) 0.001$ \\
ANA positivity, $\mathrm{n}(\%)$ & $4.50(1.02,19.83) 0.047$ & - \\
Cardiac involvement, & $2.28(1.21,4.31) 0.010$ & - \\
$\quad \mathrm{n}(\%)$ & & \\
Xerophthalmia, $\mathrm{n}(\%)$ & $1.82(1.02,3.27) 0.044$ & $4.82(1.56,14.95) 0.006$ \\
Low C3 level $(<0.9 \mathrm{~g} / \mathrm{L})$ & $1.83(1.01,3.30) 0.046$ & - \\
Saliva gland atrophy & $0.38(0.19,0.74) 0.004$ & - \\
ESR (mm/h) & - & $0.98(0.96,1.00) 0.0336$ \\
\hline
\end{tabular}

NP neurological, ESSDAl European League Against Rheumatism Sjögren's syndrome disease activity index, ESSPRI European League Against Rheumatism Sjögren's syndrome patient reported index, ANA antinuclear antibodies, C3 complement component 3, C4 complement component 4, CRP C-reactive protein, ESR erythrocyte sedimentation rate, $L S G B$ labial salivary gland biopsy, $R F$ rheumatoid factor by separate the cohort into two subgroups. There were 248 patients younger than 50 , and 318 were the opposite (including 50 years old). We found that male, low C3 level presented significant associations with neurological involvement in pSS patients who were younger than 50 year old, while ANA positivity, cardiac involvement, saliva gland atrophy were found significant in those older than 50 year old (Table 6). Xerophthalmia and low C3 level were demonstrated to be associated with neurological involvement in the younger subgroup of sSS patients, and xerophthalmia level also presented in the older subgroup (Table 6).

\section{Discussion}

This study is to investigate the neurological involvement in a Chinese SS cohort. The prevalence of peripheral and central nervous system are about 19 and $15.2 \%$ respectively in pSS, which lied in the wide ranges came from various studies $[21,22]$. However, sSS seemed to complicate with more PNS manifestations (31.1\%). Most of our sSS were secondary to SLE and RA. It's been reported the prevalence of neuropsychiatric syndromes in SLE at time of diagnosis with SLE were 28\% [23], but the estimate incidence of neurological symptoms in RA were high up to $70 \%$ when mood disorders are included [24]. In our cohort, neuropsychiatric symptoms like depression is not uncommon, which should arouse more attention. Among various manifestations of neurological involvement, limbs pain, limbs numbness and cerebral infarction were most frequently seen in more than a third of the cohort which suggesting an ischemic mechanism and

Table 6 Multivariate analysis of factors associated with neurological involvement in primary and secondary Sjögren's syndrome

\begin{tabular}{lll}
\hline Independent variables & \multicolumn{2}{l}{$\begin{array}{l}\text { Multivariate analysis } \\
\text { OR }(\mathbf{9 5} \% \mathrm{Cl}) \boldsymbol{P} \text { value }\end{array}$} \\
\cline { 2 - 3 } & pSS & \multicolumn{2}{c}{ sSS } \\
\hline Age $<50$ & & \\
Sex (male) & $4.56(1.15,20.01) 0.013$ & \\
Low C3 level $(<0.9 \mathrm{~g} / \mathrm{L})$ & $2.21(1.04,4.82) 0.026$ & $3.33(1.92,8.03) 0.021$ \\
Xerophthalmia, $\mathrm{n}(\%)$ & & $3.03(1.08,7.45) 0.030$ \\
Age $>=50$ & & \\
ANA positivity & $5.52(1.42,37.28) 0.037$ & \\
Cardiac involvement & $2.38(1.18,5.06) 0.013$ & \\
Saliva gland atrophy & $0.28(0.13,0.59) 0.001$ & \\
Xerophthalmia, $\mathrm{n}(\%)$ & & $4.07(1.25,14.35) 0.012$ \\
\end{tabular}

NP neurological, ESSDAI European League Against Rheumatism Sjögren's syndrome disease activity index, ESSPRI European League Against Rheumatism Sjögren's syndrome patient reported index, ANA antinuclear antibodies, C3 complement component 3, C4 complement component 4, CRP C-reactive protein, ESR erythrocyte sedimentation rate, $L S G B$ labial salivary gland biopsy, $R F$ rheumatoid factor 
a vasculitis process in SS. Epilepsy had been described in pSS, but is not well characterized [7]. We also found 3 cases with epilepsy as their initial symptoms and were then diagnosed by neuropathists. When compare the differences between pSS and sSS, we found the later have higher frequencies of xerostomia, articular and saliva gland involvement and low complement prevalence. Given the wide involvement of neurological involvement in SS, tight control of neurological risk factors is needed for better prognosis.

For potential risk factors for neurological involvement, low C3 level was found to be associated in younger pSS patients, and ANA positivity, cardiac involvement, saliva gland atrophy were demonstrated to be associated in elder pSS patients. It revealed that cardiac event accumulates with age and plays a key role in neurological involvement. For example, a marked increase in the risk of stroke was found after acute myocardial infarction stroke revealed its strong association with high cardiovascular risk [25]. Saliva gland atrophy was confirmed by histology of LSGB. Significant lymphocytic in filtration in the LSGB, defined as a focus score (FS) $\geq 1$, has a preponderant role in both AECG and ACR classifications and is a promising tool for prognosis [26]. It and has sensibility comparable to scintigraphy and sialography and could not be substituted by ultrasound for all patients [27]. However, the sensitivity and specificity of commonly seen saliva gland atrophy for SS remain unclear. Here, we reveal the potential relationship between saliva gland atrophy and neurological involvement in pSS, especially meaningful in the elder, while the pathogenesis to be further investigated. ANA positivity has been found to be associated with uveitis risk in Juvenile idiopathic arthritis [28] and is considered as a significant predictors of cardiovascular events and mortality in both those with and those without rheumatic diseases, which hints the its potential role in neurological involvement [29].

Furthermore, xerophthalmia were found to be associated with neurological involvement in both younger and older sSS patients, but low C3 level was only connected with those younger sSS patients. As is known, the complement system is a major component of the innate immune system, is becoming increasingly recognized as a critical participant in the acute inflammatory pathophysiology of acquired brain or spinal cord injury [30, 31]. C3 represents the central molecule of the complement cascade [32]. Low level of C3 in SS patients' serum which suggesting the excessive activation and consumption of complement system, was revealed to be an independent risk factor for neurological involvement both in pSS and sSS patients who were less than 50 years old. Our finding further supports the role of complement system in immunological associated neurological lesion. Also, the different risk factors of neurological involvement in the two age subgroups suggested distinctive mechanisms of pathogenesis behind them.

The broad spectrums of clinical profiles, different classification criteria and ethnic diversity have posed challenges to a comprehensive and accurate appraisal of SS. In our study it's demonstrated that low C3 level is a good to predictor for neurological complications in younger SS, both in primary and secondary SS, which might provide insight into better clinical decision-making, especially at early stages of the disease.

However, limitation of this study should be considered, that we included a homogenous group of participants from one center, which might provide compelling results. As it is a cross-sectional study, is limited to correlation analysis and unable to support strong causal conclusions. And traditional risk factors like arteriosclerosis, age, hypertension, therapeutic side effects, were not adjusted in the present study, which might also play a key role in the development of the neurological complications in SS. Therefore, to further evaluate the role of complement neurological complications in SS, more data from heterogeneous SS patients with consecutive follow-up are highly recommended.

\section{Conclusions}

Low complement (C3) levels, xerophthalmia, ANA positive, cardiac involvement and labial salivary gland histological result were good ways to predict neurological complications in different subgroups of SS, which might provide insight into better clinical decision-making, especially at early stages of the disease.

\section{Abbreviations \\ SS: Sjögren's syndrome; sSS: secondary Sjögren's syndrome; pSS: primary Sjögren's syndrome; PNS: peripheral nervous system; C3/C4: complement 3/ complement 4; CNS: central nervous system; LA: leukoaraiosis; LSGB: labial salivary gland biopsy; ESR: erythrocyte sedimentation rate; CRP: C-reactive protein; RF: rheumatoid factor; ANA: antinuclear antibodies; EULAR: European League Against Rheumatism; ESSDAI: European League Against Rheumatism Sjögren's syndrome disease activity index; ESSPRI: European League Against Rheumatism Sjögren's syndrome patient reported index.}

\section{Authors' contributions}

XSH, WQ and TZ collected the clinical data of patients that enrolled in this study. XFZ and XCZ made substantial contributions to conception and design, and analyzed the patient data regarding the rheumatic disease. SYC assessed the nervous system involvement in SS patients and analyzed data regarding nervous system disease. CXL assessed the oral symptoms in SS patient. All authors involved in drafting the manuscript. WJY and XBW was the major contributor in revising the manuscript and given final approval of the version to be published. All authors agreed to be accountable for all aspects of the work in ensuring that questions related to the accuracy or integrity of any part of the work are appropriately investigated and resolved. All authors read and approved the final manuscript.

\section{Author details}

${ }^{1}$ Rheumatology Department, The First Affiliated Hospital of Wenzhou Medical University, Wenzhou, China. ${ }^{2}$ Rheumatology Department, Ruian People's 
Hospital, Wenzhou, China. ${ }^{3}$ Neurology Department, The First Affiliated Hospital of Wenzhou Medical University, Wenzhou, China. ${ }^{4}$ Stomatological Department, The First Affiliated Hospital of Wenzhou Medical University, Wenzhou, China

\section{Acknowledgements}

We thank the patients for participating in this study. We also thank Professor Jianmin Li for providing expert advice on salivary gland pathology.

\section{Competing interests}

The authors declare that they have no competing interests.

\section{Availability of data and materials}

The datasets used and/or analysed during the current study are available from the corresponding author on reasonable request.

\section{Consent for publication}

Not applicable.

\section{Ethics approval and consent to participate}

This study was approved by the Ethical Committee of the First Affiliated Hospital of Wenzhou Medical University (approval \# 16024). The study design conformed to current National Health and Family Planning Commission of China ethical standards, with written informed consent provided by all patients.

\section{Funding}

No funding was obtained for this study.

\section{Publisher's Note}

Springer Nature remains neutral with regard to jurisdictional claims in published maps and institutional affiliations.

Received: 15 October 2017 Accepted: 19 April 2018

Published online: 27 April 2018

\section{References}

1. Moreira I, Teixeira F, Silva AM, Vasconcelos C, Farinha F, Santos E. Frequent involvement of central nervous system in primary Sjögren syndrome. Rheumatol Int. 2015;35(2):289-94.

2. Vitali C, Bombardieri S, Moutsopoulos HM, Balestrieri G, Bencivelli W Bernstein RM, Bjerrum KB, Braga S, Coll J, Vita SD. Preliminary criteria for the classification of Sjögren's syndrome. Results of a prospective concerted action supported by the European Community. Arthritis Rheum. 1993:36(3):340-7.

3. Mavragani CP, Moutsopoulos HM. The geoepidemiology of Sjogren's syndrome. Autoimmun Rev. 2010;9(5):A305-10.

4. Fox Rl. Sjogren's syndrome. Lancet. 2005;366(9482):321-31.

5. Ramos Casals M, Brito Zerón P, Sisó Almirall A, Bosch Aparici FJ. Primary Sjogren syndrome. Br Med J. 2012;344:e3821.

6. Alexander EL, Provost TT, Stevens MB, Alexander GE. Neurologic complications of primary Sjogren's syndrome. Medicine (Baltimore). 1982;61(4):247-57.

7. Delalande S, de Seze J, Fauchais AL, Hachulla E, Stojkovic T, Ferriby D, Dubucquoi S, Pruvo JP, Vermersch P, Hatron PY. Neurologic manifestations in primary Sjogren syndrome: a study of 82 patients. Medicine (Baltimore). 2004;83(5):280-91.

8. Vitali C, Bombardieri S, Jonsson R, Moutsopoulos H, Alexander E, Carsons S, Daniels T, Fox P, Fox R, Kassan S. Classification criteria for Sjögren's syndrome: a revised version of the European criteria proposed by the American-European Consensus Group. Ann Rheum Dis. 2002;61 (6):554-8.

9. Segal B, Carpenter A, Walk D. Involvement of nervous system pathways in primary Sjogren's syndrome. Rheum Dis Clin North Am. 2008;34(4):885906 , viii.

10. Le Guern V, Belin C, Henegar C, Moroni C, Maillet D, Lacau C, Dumas JL, Vigneron N, Guillevin L. Cognitive function and 99mTc-ECD brain SPECT are significantly correlated in patients with primary Sjogren syndrome: a case-control study. Ann Rheum Dis. 2010;69(1):132-7.
11. Tan EM, Cohen AS, Fries JF, Masi AT, Mcshane DJ, Rothfield NF, Schaller JG, Talal N, Winchester RJ. The 1982 revised criteria for the classification of systemic lupus erythematosus. Arthritis Rheum. 1982;25(11):1271-7.

12. Masi AT. Preliminary criteria for the classification of systemic sclerosis (scleroderma). Arthritis Rheum. 1980;23(5):581-90.

13. Aletaha D, Neogi T, Silman AJ, Funovits J, Felson DT, Bingham CO, Birnbaum NS, Burmester GR, Bykerk VP, Cohen MD. 2010 rheumatoid arthritis classification criteria: an American College of Rheumatology/European League Against Rheumatism collaborative initiative. Arthritis Rheum. 2010;62(9):2569-81.

14. Bohan A, Peter JB. Polymyositis and dermatomyositis. N Engl J Med. 1975;292(8):403-7.

15. Sharp GC, Anderson PC. Current concepts in the classification of connective tissue diseases: overlap syndromes and mixed connective tissue disease (MCTD). J Am Acad Dermatol. 1980;2(4):269-79.

16. Kaplan MM, Gershwin ME. Primary biliary cirrhosis. N Engl J Med. 2005;353(12):1261-73.

17. Guellec D, Cornec D, Jousse-Joulin S, Marhadour T, Marcorelles P, Pers J-O, Saraux A, Devauchelle-Pensec V. Diagnostic value of labial minor salivary gland biopsy for Sjögren's syndrome: a systematic review. Autoimmun Rev. 2013;12(3):416-20.

18. Goren MB, Goren SB. Diagnostic tests in patients with symptoms of keratoconjunctivitis sicca. Am J Ophthalmol. 1988;106(5):570-4.

19. Whitcher JP, Shiboski CH, Shiboski SC, Heidenreich AM, Kitagawa K, Zhang S, Hamann S, Larkin G, McNamara NA, Greenspan JS. A simplified quantitative method for assessing keratoconjunctivitis sicca from the Sjögren's Syndrome International Registry. Am J Ophthalmol. 2010;149(3):405-15.

20. Risselada AP, Kruize AA, Bijlsma JW. Clinical applicability of the EULAR Sjogren's syndrome disease activity index: a cumulative ESSDAI score adds in describing disease severity. Ann Rheum Dis. 2012;71(4):631.

21. Font J, Ramoscasals M, de la Red G, Pou A, Casanova A, García-Carrasco M, Cervera R, Molina JA, Valls J, Bové A, Ingelmo M, Graus F. Pure sensory neuropathy in primary Sjogren's syndrome. Longterm prospective followup and review of the literature. J Rheumatol. 2003;30(7):1552.

22. Bougea A, Anagnostou E, Konstantinos G, George P, Triantafyllou N, Kararizou E. A systematic review of peripheral and central nervous system involvement of rheumatoid arthritis, systemic lupus erythematosus, primary Sjögren's syndrome, and associated immunological profiles. Int J Chronic Dis. 2015;2015(6):1-11.

23. Muscal E, Brey RL. Neurological manifestations of systemic lupus erythematosus in children and adults. Neurol Clin. 2010;28(1):61-73.

24. Covic T, Cumming SR, Pallant JF, Manolios N, Emery P, Conaghan PG, Tennant A. Depression and anxiety in patients with rheumatoid arthritis: prevalence rates based on a comparison of the Depression, Anxiety and Stress Scale (DASS) and the hospital, Anxiety and Depression Scale (HADS). BMC Psychiatry. 2012;12:6.

25. Hachet O, Guenancia C, Stamboul K, Daubail B, Richard C, Bejot Y, Yameogo V, Gudjoncik A, Cottin Y, Giroud M, Lorgis L. Frequency and predictors of stroke after acute myocardial infarction: specific aspects of in-hospital and postdischarge events. Stroke. 2014;45(12):3514-20.

26. Milic VD, Petrovic RR, Boricic IV, Marinkovic-Eric J, Radunovic GL, Jeremic PD, Pejnovic NN, Damjanov NS. Diagnostic value of salivary gland ultrasonographic scoring system in primary Sjogren's syndrome: a comparison with scintigraphy and biopsy. J Rheumatol. 2009;36(7):1495-500.

27. Song GG, Lee YH. Diagnostic accuracies of sialography and salivary ultrasonography in Sjogren's syndrome patients: a meta-analysis. Clin Exp Rheumatol. 2014;32(4):516.

28. Heiligenhaus A, Heinz C, Edelsten C, Kotaniemi K, Minden K. Review for disease of the year: epidemiology of juvenile idiopathic arthritis and its associated uveitis: the probable risk factors. Ocul Immunol Inflamm. 2013;21(3):180-91.

29. Liang KP, Kremers HM, Crowson CS, Snyder MR, Therneau TM, Roger VL, Gabriel SE. Autoantibodies and the risk of cardiovascular events. J Rheumatol. 2009;36(11):2462-9.

30. Neher MD, Weckbach S, Flierl MA, Huber-Lang MS, Stahel PF. Molecular mechanisms of inflammation and tissue injury after major trauma-is complement the "bad guy"? J Biomed Sci. 2011;18:90. 
31. Brennan FH, Anderson AJ, Taylor SM, Woodruff TM, Ruitenberg MJ. Complement activation in the injured central nervous system: another dual-edged sword? J Neuroinflammation. 2012;9:137.
32. Ricklin D, Hajishengallis G, Yang K, Lambris JD. Complement: a key system for immune surveillance and homeostasis. Nat Immunol. 2010;11(9):785-97.

- fast, convenient online submission

- thorough peer review by experienced researchers in your field

- rapid publication on acceptance

- support for research data, including large and complex data types

- gold Open Access which fosters wider collaboration and increased citations

- maximum visibility for your research: over 100M website views per year

At BMC, research is always in progress.

Learn more biomedcentral.com/submissions 